Proceedings of the 2010 Winter Simulation Conference

B. Johansson, S. Jain, J. Montoya-Torres, J. Hugan, and E. Yücesan, eds.

\title{
A SPECTRUM OF TRAFFIC FLOW MODELING AT MULTIPLE SCALES
}

\author{
Daiheng Ni \\ Development of Civil and Environmental Engineering \\ University of Massachusetts Amherst \\ MA 01003, U.S.A.
}

\begin{abstract}
This paper presents a broad perspective on traffic flow modeling at a spectrum of four scales. Modeling objectives and model properties at each scale are discussed and existing efforts are reviewed. In order to ensure modeling consistency and provide a microscopic basis for macroscopic models, it is critical to address the coupling among models at different scales, i.e. how less detailed models are derived from more detailed models and, conversely, how more detailed models are aggregated to less detailed models. With this understanding, a consistent modeling approach is proposed based on field theory and modeling strategies at each of the four scales are discussed. In addition, a special case is formulated at both microscopic and macroscopic scales. Numerical and empirical results suggest that these special cases perform satisfactorily and aggregate to realistic macroscopic behavior. By ensuring model coupling and modeling consistency, the proposed approach is able to establish the theoretical foundation for traffic modeling and simulation at multiple scales seamlessly within a single system.
\end{abstract}

\section{INTRODUCTION}

Anyone who used maps probably developed the following experience. Fifteen years ago, a 1:10,000 paper map was needed to view a city (e.g. Amherst, MA), while a 1:1,000,000 paper map was needed to view a state (e.g. Massachusetts). If the scale was changed, a new map was needed. Today, using digital maps (e.g. Google maps), one is able to overview the entire country, and then progressively zoom in to view Massachusetts, Amherst, and even the UMass Amherst campus, all seamlessly and within a single system.

Similarly, it is desirable that traffic simulation would allow an analyst to zoom in to examine low-level details and zoom out to overview system-wide performance within the same simulation process. Figure 1 illustrates such a paradigm. The background represents a macroscopic view of traffic operation in an entire region. This is analogous to viewing traffic 10,000 $\mathrm{m}$ above the ground and the traffic appears to be a compressible fluid whose states (speed, flow, and density, etc.) propagate like waves. As one zooms in to a local area of the region, a mesoscopic view is obtained. This is like viewing traffic 3,000 $\mathrm{m}$ above the ground where the sense of waves recedes and a scene of particles emerges. As one further zooms in to a segment of the roadway, a microscopic view is resulted. Similar to watching traffic $1,000 \mathrm{~m}$ above the ground, the scene is dominated by moving particles that interact with each other so as to maintain safe positions in traffic stream. Finally, if one focuses on a few neighboring vehicles, a picoscopic view is achieved as if one were operating one of the vehicles. As such, one has to interact with the driving environment (e.g. roadway, signs, signals, etc.), make control decisions, and manage vehicle dynamic respond to travel safely. If such a "zoomable" simulation becomes available, one would be able to translate traffic flow representation among multiple scales, 
e.g. to trace a low-level event all the way to a high-level representation and, conversely, to decompose a global problem down to one or more local deficiencies. As such, the "zoomable" simulation will transform the way that traffic flow is analyzed and transportation problems are addressed.

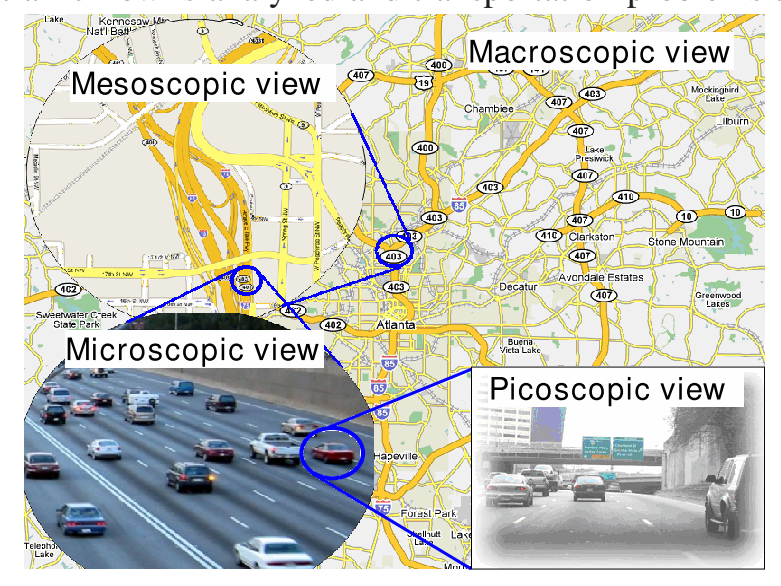

Figure 1: Multiscale traffic flow modeling

The objective of this paper is to address multiscale traffic flow modeling with inherent consistency. The term consistency here concerns the coupling among models at different scales, i.e. how less detailed models are derived from more detailed models and, conversely, how more detailed models are aggregated to less detailed models. Only consistent multiscale models are able to provide the theoretical foundation for the above "zoomable" traffic simulation.

\section{THE SPECTRUM OF MODELING SCALES}

The modeling of traffic flow can be performed at, but is not limited to, a spectrum of four scales, namely picoscopic, microscopic, mesoscopic, and macroscopic from the most to the least detailed in that order. Considering that the definition of these modeling scales are rather vague, implicit, or absent in the literature, this section attempts to provide an explicit definition so that existing and future models are easily classified and related. Such a definition is tabulated in Figure 2 for each of the four modeling scales based on their properties (i.e. rows in the table). The first three rows ("State variable", "Variable description", and "State diagram") are discussed in this section and the remaining three rows ("Underlying principle", "Modeling approach", and "Model coupling") pertain to the proposed multiscale approach with inherent consistency which are to be elaborated in the next section.

\subsection{The Picoscopic Scale}

Picoscopic modeling should be able to represent traffic flow so that the trajectory of each vehicle, $\left(x_{i}(t), y_{i}(t)\right)$ where $i \in\{1,2,3, \ldots, I\}$ denotes vehicle ID, can be tracked in both longitudinal $x$ and lateral $y$ directions over time $t \geq 0$. Knowing these vehicle trajectories, the state and dynamics of the traffic system can be completely determined. Therefore, $\left(x_{i}(t), y_{i}(t)\right)$ is the state variable (one or a set of variables that characterizes the state of a system). The corresponding state diagram (a graphical representation that illustrates the dynamics or evolution of system state) consists of these vehicle trajectories in a three-dimensional domain $(x, y, t)$. Picoscopic models are mainly of interest in automotive engineering. Dynamic vehicle models with varying degrees of freedom have been proposed (Abe 1985, Antoun et al. 1986). A myriad of driver models have been reported to assist various aspects of automotive engineering including vehicle handling and stability (Wierwille, Gagne, and Knight 1967, Blaauw, Godthelp, and Milgram 1984). 


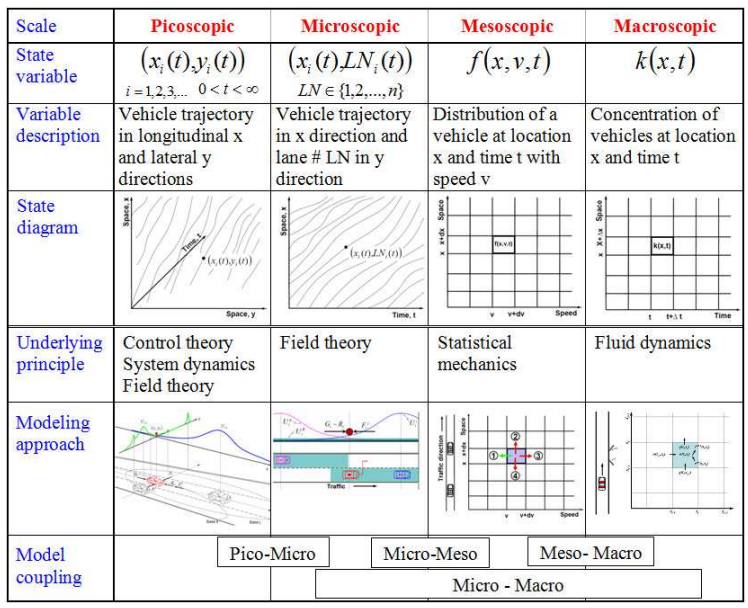

Figure 2: The spectrum of modeling scales

\subsection{The Microscopic Scale}

Microscopic modeling should be able to represent traffic flow so that the trajectory of each vehicle can be tracked in the longitudinal direction $x_{i}(t)$ with the lateral direction being discretized by lanes $L N_{i}(t)$ where $L N \in\{1,2, \ldots, n\}$. Hence, $\left(x_{i}(t), L N_{i}(t)\right)$ is state variable that describe the sate and dynamics of traffic flow at this scale and the corresponding state diagram consists of vehicle trajectories in a two-dimensional domain $(x, t)$. Within traffic flow community, microscopic models treat drivervehicle units as massless particles with personalities. The behavior of these particles is governed by car-following models (Chandler, Herman, and Montroll 1958, Gazis, Herman, and Rothery 1961) in the longitudinal direction and discrete choice (e.g. lane-changing and gap-acceptance) models (Gipps 1986, Ahmed et al. 1996, Raff and Hart 1950, Velan and Van Aerde 1996) in the lateral direction. Surveys on microscopic models can be found in the literature(Gartner, Messer, and Rathi 2001, Hoogendoorn and Bovy 2001).

\subsection{The Mesoscopic Scale}

Mesoscopic modeling should be able to represent traffic flow so that the probability of the presence of a vehicle at a longitudinal location $x$ with speed $v$ at time $t$ is tracked. The lateral direction is only of interest if it provides passing opportunities. The state diagram typically involves a two-dimensional domain $(x, v)$ at an instant $t$ and the domain is partitioned into cells with space increment $d x$ and speed increment $d v$. The state variable is a distribution function $f(x, v, t)$ such that $f(x, v, t) d x d v$ denotes the probability of having a vehicle within space range $(x, x+d x)$ and speed range $(v, v+d v)$ at time $t$. Knowing the distribution function $f(x, v, t)$, the dynamics of the system can be determined statistically. Conventional mesoscopic traffic flow models come with three flavors. First, models such as the one in TRANSIMS (Smith et al. 1995, Morrison and Loose 1995, LANL 1999) take a Cellular Automata approach. Second, models such as those implemented in DynaMIT (Ben-Akiva et al. 1998) and DYNASMART (Chang, Junchaya, and Santiago 1994) use macroscopic models (such as speeddensity relationship), as oppose to microscopic car-following models, to determine vehicle speed and movement. Third, truly mesoscopic models such as the one postulated by Prigogine and his co-workers (Prigogine and Andrews 1960) are based on non-equilibrium statistical mechanics or kinetic theory which draw analogy between classical particles and highway vehicles. 


\subsection{The Macroscopic Scale}

Macroscopic modeling should be able to represent traffic flow so that only local aggregation of traffic flow (e.g. density $k$, speed $u$, and flow $q$ ) over space (longitudinal) $x$ and time $t$ is tracked. Traffic density $k(x, t)$ is a good candidate of state variable because, unlike flow and speed, density is an unambiguous indicator of traffic condition. The state diagram typically involves a two dimensional domain $(x, t)$. Knowing $k(x, t)$, the dynamics of the system can be determined macroscopically. Conventional macroscopic traffic flow models describe the propagation of traffic disturbances as waves. A fundamental basis for formulating wave propagation is the law of conservation. The firstorder form of the law is mass/vehicle conservation (Lighthill and Whitham 1955, Richards 1956). A model is of a higher order if it incorporates other forms of conservation (Payne 1971, Whitham 1974). Surveys of macroscopic models can be found in the literature (Gartner, Messer, and Rathi 2001).

\subsection{Issues of Multiscale Modeling}

Remarkably, existing models at the same scale typically follow different modeling approaches and, hence, it is difficult to relate these models to each other. In addition, models at different modeling scales are rarely coupled. For example, a macroscopic model typically lacks a microscopic basis and a microscopic model does not have its macroscopic counterpart.

Therefore, an ideal multiscale modeling approach should emphasize not only model quality at each individual scale but also the coupling between different scales. Only models formulated following such an approach is able to support the "zoomzble" traffic simulation discussed in Section 1. As such, the resulting state diagram at a more detailed scale contains the necessary information to reproduce a less detailed diagram, as illustrated in Figure 2. For example, the microscopic diagram is simply a projection of the picoscopic diagram onto the $x-t$ plane and the macroscopic state diagram can be completely reconstructed from the microscopic diagram using Eddie's definition of traffic flow characteristics (Eddie 1963, Ni 2007).

\section{THE PROPOSED MULTISCALE APPROACH}

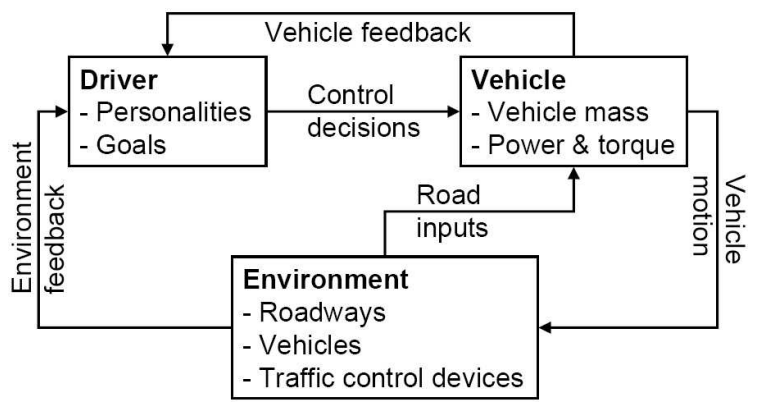

Figure 3: The closed-loop system

The objective of this section is to pursue the above multiscale modeling approach and develop strategies to formulate a spectrum of models with inherent consistency. The approach starts at the picoscopic scale by formulating a model that is mathematically amenable to representing the natural way of human thinking while comply to physical principles; the microscopic model can be simplified from the picoscopic model yet still capturing the essential mechanisms of vehicle motion and interaction; the mesoscopic model can be derived from the microscopic model based on principles of non-equilibrium statistical mechanics; the macroscopic model can be derived from the mesoscopic model by applying principles of fluid dynamics. See a summary of underlying principle, modeling approach, and modeling coupling in Figure 2. 


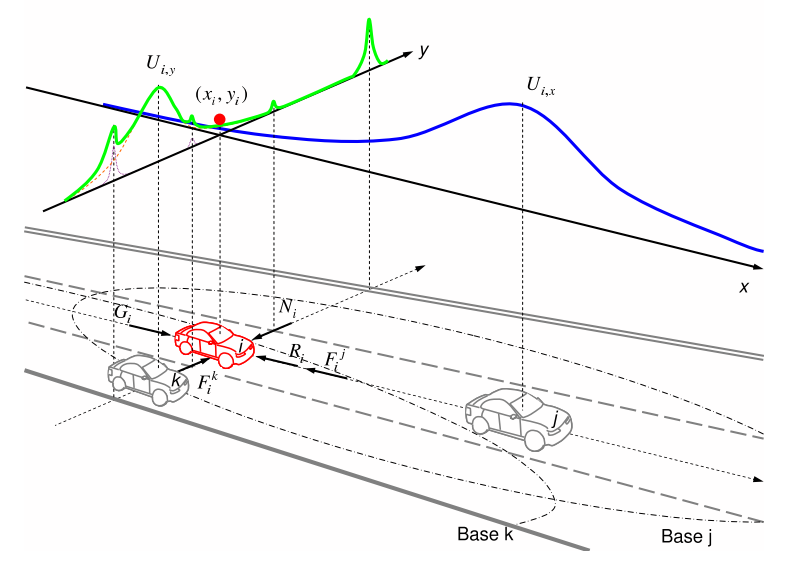

Figure 4: The illustration of a perceived field

\subsection{Picoscopic modeling}

In order to conform to real-world driving experiences, the proposed model should mimic the way that a driver operates his/her vehicle and responds to the driving environment. Based on principles of control theory, a driver-vehicle-environment closed-loop control (DVECLC) system (Ni 2006) has been developed. Figure 3 illustrates the components of the system and its control flow including feedback loops.

This system consists of a driver model and a vehicle model which interact with each other as well as with the driving environment. the driver receives information from the environment such as roadways, traffic control devices, and the presence of other vehicles. The driver also receives information from his/her own vehicle such as speed, acceleration, and yaw rate. These sources of information, together with driver properties and goals, are used to determine driving strategies (such as steering and gas/brake). The driving strategies are fed forward to the vehicle which also receives input from roadways. These sources of information, together with vehicle properties, determine the vehicle's dynamic responses based on vehicle dynamic equations. Moving longitudinally and laterally, the vehicle constitute part of the environment. Other vehicle dynamic responses such as speed, acceleration, and yaw rate are fed back to the driver for determining driving strategies in the next step. Thus traffic operation is the collection of movement and interaction of all vehicles in the environment.

Assuming a long section of highway without worrying about routing and turning, the driver model can be formulated by applying principles of field theory. Basically, objects in a traffic system (e.g. roadways and vehicles) are perceived by a subject driver as component fields. The driver interacts with an object at a distance and the interaction is mediated by the field associated with the object. The superposition of these component fields represents the overall hazard encountered by the subject driver. Hence, the objective of vehicle motion is to seek the least hazardous route by navigating through the field along its valley and traffic flow consists of the motion and interaction of all vehicles. With this understanding, the driver model at the picoscopic scale is formulated as follows. Figure 4 illustrates a scenario where the subject driver-vehicle unit $i$ (the trailing one in the middle lane) is moving along with its leader $j$ in the same lane and neighbor $k$ in the adjacent lane. The overall field perceived by driver $i$ is denoted as $U_{i}$ whose two cross sections $U_{i, x}$ and $U_{i, y}$ are shown above the ground with vehicle $i$ illustrated as a dot riding on the field. The vehicle is subject to forces from the roadway and surrounding vehicles. Such forces can be interpreted as the stress on the driver to pursue desired speed $v_{i}$, keep lane, and observe safe distances. More specifically, these forces include: roadway gravity $G_{i}$ which accelerates the vehicle, roadway resistance $R_{i}$ which prevents the vehicle from speeding, lane keeping force $N_{i}$ should the vehicle depart its current lane, vehicle 


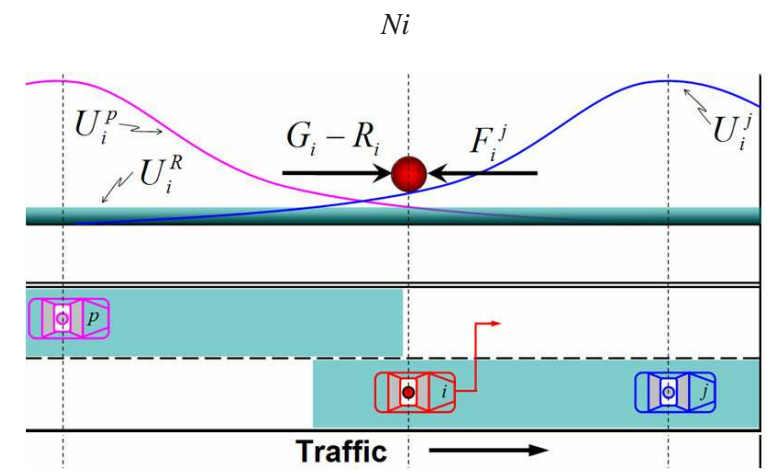

Figure 5: Microscopic modeling

interaction forces $F_{i}^{j}$ and $F_{i}^{k}$ with the former preventing forward crash and the latter side crash. By applying principles of field theory, the following can be established $F_{i}^{j}=\frac{U_{i, x}}{x}$ and $F_{i}^{k}-N_{i}=-\frac{U_{i, y}}{y}$. Therefore, by incorporating time $t$ and perception-reaction time $i$, the net force acting on driver $i$ can be formulated as:

$$
\left\{\begin{array}{l}
m_{i} \ddot{x}_{i}(t+i)=F_{i, x}(t)=G_{i}(t)-R_{i}(t)-F_{i}^{j}(t)=G_{i}(t)-R_{i}(t)+\frac{U_{i, x}}{x} \\
m_{i} \ddot{y}_{i}(t+i)=F_{i, y}(t)=F_{i}^{k}-N_{i}=-\frac{U_{i, y}}{y}
\end{array}\right.
$$

where specific forms of $G_{i}, R_{i}, F_{i}^{j}, F_{i}^{k}, N_{i}$, and $U_{i}$ are open for admitting different modeling strategies. This equation system determines driver $i$ 's operational control in longitudinal and lateral directions.

\subsection{Microscopic modeling}

The microscopic model can be formulated by simplifying the above picoscopic model as follows: (a) ignoring interactions inside a driver-vehicle unit allowing it to be modeled as an active particle, (b) representing a driver's longitudinal and lateral control using separate but simpler models, (c) reducing the vehicle dynamic system to a particle, and (d) simplifying roadway surfaces to a collection of lines.

With the above simplifications, the two-dimensional (3D) potential field $U$ in Figure 4 reduces to a $2 \mathrm{D}$ potential function. The upper part of Figure 5 illustrates an example where a subject driver $i$ (the middle one) is traveling behind a leading vehicle $j$ and followed by a third vehicle $p$ in the adjacent lane. The potential field $U_{i}$ perceived by the driver is shaded in the lower part of the figure and is represented by a curve in the upper part. Since the trailing vehicle in the adjacent lane does not affect the subject driver's longitudinal motion, the "stress" on the subject driver to keep safe distance only comes from the leading vehicle and can be represented as: $F_{i}^{j}=-\frac{U_{i}^{j}}{x}$. By incorporating roadway gravity $G_{i}$, roadway resistance $R_{i}$, and interaction between vehicles $F_{i}^{j}$, the net force on $i$ can be expressed more specifically as: $m_{i} \ddot{x}_{i}=G_{i}-R_{i}-F_{i}^{j}$. Departing from the above equation, a special case deserves particular attention:

$$
\ddot{x}_{i}\left(t+{ }_{i}\right)=g_{i}\left[1-\left(\frac{\dot{x}_{i}(t)}{v_{i}}\right)-e^{\frac{s_{i j}(t)^{*}-s_{i j}(t)}{s_{i j}(t)^{*}}}\right]
$$

where it is assumed that $G_{i}=m_{i} \times g_{i}, R_{i}=m_{i} \times\left(\frac{\dot{x}_{i}(t)}{v_{i}}\right), F_{i}^{j}=m_{i} \times f\left(s_{i j}, s_{i j}(t)^{*}\right), m_{i}$ is vehicle mass, $g_{i}$ is the maximum acceleration that driver $i$ is willing to apply when starting from stand still, $\dot{x}_{i}(t)$ is the actual speed of vehicle $i, v_{i}$ is the desired speed of driver $i, s_{i j}=x_{j}-x_{i}$ is the actual spacing between vehicles $i$ and $j, x_{i}$ is the position of vehicle $i, x_{j}$ is the position of vehicle $j, s_{i j}^{*}$ is the desired spacing between vehicles $i$ and $j . L_{j}$ is the nominal length of vehicle $j$ and is conveniently used as 


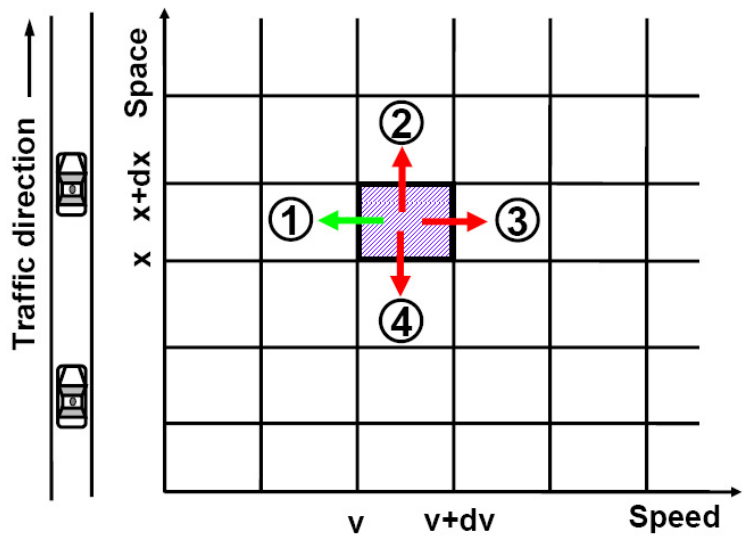

Figure 6: The $\mathrm{x}-\mathrm{v}$ diagram

the spacing between two vehicles in jammed traffic. The the difference $\left(s_{i j}^{*}-s_{i j}\right)$ represents how far vehicle $i$ intrudes beyond $s_{i j}^{*}$. The rationale of representing the interaction force $F_{i}^{j}$ between vehicles $i$ and $j$ using an exponential function is to set the desired spacing $s_{i j}^{*}$ as a base line, beyond which the intrusion by unit $i$ is translated exponentially to the repelling force acting on the unit. The desired spacing $s_{i j}^{*}$ can be further determined as follows. According to (Gipps 1981), the desired spacing should allow vehicle $i$ to stop behind its leading vehicle $j$ after a perception-reaction time $i$ and a deceleration process at a comfortable level $b_{i}>0$ should vehicle $j$ applies an emergency brake at rate $B_{j}>0$. This rule results in: $s_{i j}^{*}(t)=x_{i-1}(t)-x_{i}(t) \geq \frac{\dot{x}_{i}^{2}(t)}{2 b_{i}}+\dot{x}_{i}{ }_{i}-\frac{\dot{x}_{i-1}^{2}(t)}{2 B_{i-1}}+L_{j}$.

The driver's lateral control concerns changing lanes to seek a speed gain or to use an exit. The shaded areas in the bottom part of Figure 5 can be interpreted as drivers $j$ and $p$ 's personal spaces. A lane change decision is reached whenever driver $i$ intrudes into another driver's personal space. With such a decision, driver $i$ begins to search for open spaces in adjacent lanes. In this particular case, such an open space happens to be available in the left lane barely allowing the center of vehicle $i$ to move in. Consequently, the result of the gap acceptance decision is to abruptly switch vehicle $i$ to the left lane.

\subsection{Mesoscopic modeling}

Mesoscopic modeling applies principles of Non-Equilibrium Statistical Mechanics or kinetic theory to model traffic flow. Essential to the modeling is the determination of a distribution function $f(x, v, t)$ such that $f(x, v, t) d x d v$ denotes the probability of having a vehicle within space range $(x, x+d x)$ and speed range $(v, v+d v)$ at time $t$ (see Figure 6). The time evolution of traffic flow is described by an evolution equation: $\frac{d f}{d t}=\frac{f}{t}+\frac{f}{x} \frac{d x}{d t}$ whose right-hand side is to be determined. Therefore, the central question is how to rigorously derive the evolution equation. This can be done by following a procedure similar to deriving the Boltzmann equation (Tolman 1980) from basic principles. The classical Boltzmann equation describes particles moving in a 3D domain, so the first step is to reduce the 3D case to a 1D case which represents traffic moving on a unidirectional highway.

Existing models, in particular those based on Prigogine's work, are postulated. In order to derive the 1D Boltzmann equation from basic principles, a sound understanding of the mechanism of traffic evolution is required. Existing models, including a derived model (Wegener and Klar 1996, Klar and Wegener 1999), assumed that the mechanism is vehicle "collision". For example, the fast follower $i$ in the left panel of Figure 7) keeps its speed up to the collision point and then abruptly changes its speed. To be realistic, the speed change of vehicle $i$ needs to be smooth as it approaches its leader $j$ as illustrated in the right panel of Figure 7). This is possible only if car following is incorporated as 


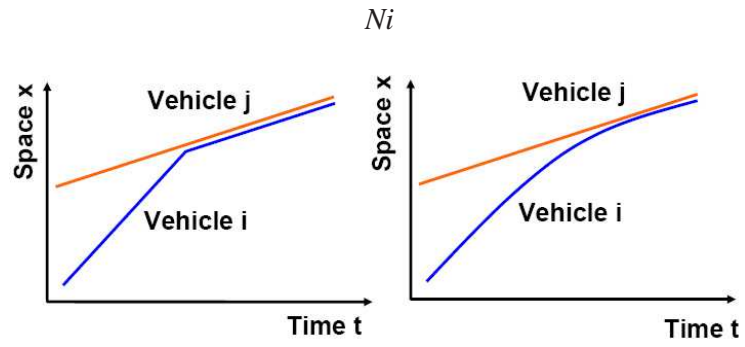

Figure 7: Car following

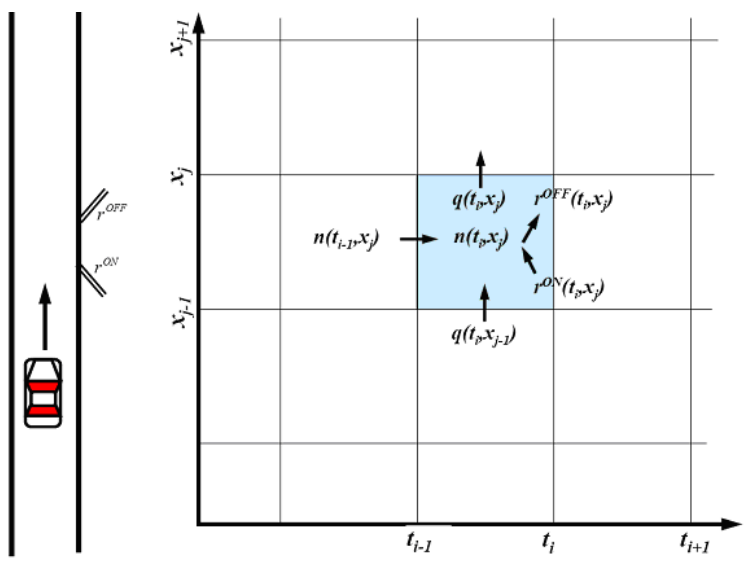

Figure 8: The finite difference method

the mechanism of particle interaction. As such, the longitudinal control model presented above can be used to derive the 1D Boltzmann equation and, thus, ensures micro-meso coupling.

The derivation of the 1D Boltzmann equation starts by applying conservation law (e.g vehicles entering and exiting the highlighted cell in Figure 6 should be conserved). Existing models considered only one direction (i.e. direction 1 below) in which vehicles exit the cell, and a similar treatment applies to vehicles entering the cell. This approach causes modeling errors. Actually, vehicles may exit the cell in four directions: (1) vehicles slowed down (and hence exited the cell) due to a sluggish leader, (2) vehicles physically moved out of the cell, (3) vehicles accelerated by an aggressive follower, and (4) vehicles reversed, which is unlikely. The opposite applies to vehicles entering the cell. Therefore, applying the law to include all directions is the correct approach. Since deriving the 1D Boltzmann equation is mathematically complicated, this paper only presents potential directions of exploration, leaving the actual derivation to be addressed in future research.

Once the 1D Boltzmann equation is formulated, one may solve it based on initial and boundary conditions to study how traffic evolves over time and space. However, solving the equation can be quite involved, as is the case for any classical Boltzmann equation. Fortunately, some important results can be inferred without fully solving the equation. For example, a hydrodynamical formulation, which is essential to macroscopic modeling, can be derived from the equation. In addition, the equation contains an equilibrium relationship between vehicle speed and traffic density, which is also essential to macroscopic modeling. Such a relationship is analogous to the Maxwell-Boltzmann distribution (the distribution of molecular speed under different temperature) which is the stationary (i.e. $\frac{f}{t}=0$ ) solution to a classical Boltzmann equation. 
3.4 Macroscopic modeling

Macroscopic modeling applies principles of Fluid Dynamics to model traffic flow as a 1D compressible continuum fluid. While the above mesoscopic modeling describes the distribution of vehicles in a highway segment, macroscopic modeling represents only the average state. Therefore, traffic density $k(x, t)$ can be related to the distribution $f(x, v, t)$ as its zeroth moment $k(x, t)=\int f(x, v, t) d v$ and traffic speed as the first moment $u(x, t)=\frac{1}{k} \int v f(x, v, t) d v$. Based on this understanding, it becomes clear that it is feasible to derive a hydrodynamical formulation from the mesoscopic model. The 1D Boltzmann equation discussed above can be expressed in a general form as: $\frac{f}{t}+v \frac{f}{x}=C$ where $C$ denotes the rate of change of $f(x, v, t)$. Multiplying both sides of this equation by $1, v$, and $\frac{1}{2} v^{2}$ and integrating over $v$ will give hydrodynamical equations of mass, momentum, and energy conservation. The mass conservation equation: $\frac{k}{t}+\frac{(k u)}{x}=\int C d v$ is of particular interest because it describes the time evolution of traffic density $k(x, t)$.

In order to solve the equation, an equilibrium speed-density relationship must be introduced into the macroscopic model. Such a relationship can be derived from the mesoscopic model under stationary conditions or, alternatively, obtained directly from the microscopic model by assuming equilibrium conditions. Presented below is an equilibrium v-k relationship derived from the special case of the microscopic model:

$$
v=v_{f}\left[1-e^{1-\frac{k^{*}}{k}}\right]
$$

where $k^{*}=v e^{-\frac{v}{v_{f}}}+\frac{1}{k_{j}}, v_{f}$ is free-flow speed, $k_{j}=1 / L, L$ is the bumper-to-bumper distance between vehicles when traffic is jammed, is average perception-reaction time of drivers. Therefore, the macroscopic model consists of a system of equations including the hydrodynamical formulation and the $\mathrm{v}-\mathrm{k}$ relationship.

$$
\begin{cases}\frac{k}{t}+\frac{(k u)}{x} & =\int C d v \\ v & =V(k)\end{cases}
$$

The the system of equations can be solved using a finite difference method, which is illustrated in Figure 8. In this method, one partitions the time-space domain into cells and keeps track of traffic flowing into and out of each cell.

\section{EMPIRICAL AND NUMERICAL RESULTS}

This section provides some empirical and numerical evidences in support of the proposed multiscale approach. Particular attention is devoted to microscopic modeling especially its special case proposed in Subsection 3.2 and its corresponding speed-density relationship presented in Subsection 3.4.

At the microscopic level, the emphasis is to check if a model makes sense since it is unrealistic to expect the same result out of a simulated run and a real one due to randomness. A physically meaningful scenario is set up as follows which consists of multiple regimes typically encountered during driving. The scenario involves two vehicles, a leader and a follower. The leader moves according to predetermined rules, while the motion of the follower is stipulated by a microscopic model. More specifically, the follower $i$ is initially stand still at $x_{i}(0)=0$. The motion of the leader $j$ is defined as follows: 


$$
\begin{cases}x_{j}=5000, \dot{x}_{j}=0, \ddot{x}_{j}=0 & \text { when } 0 \leq t<100 \\ x_{j}=2800, \dot{x}_{j}=25 & \text { when } t=100 \\ \ddot{x}_{j}=0 & \text { when } 100 \leq t<200 \\ \ddot{x}_{j}=2 & \text { when } 200 \leq t<210 \\ \ddot{x}_{j}=0 & \text { when } 210 \leq t<300 \\ \ddot{x}_{j}=-3 & \text { when } 300 \leq t<315 \\ \ddot{x}_{j}=0 & \text { when } t \geq 315\end{cases}
$$

where time $t$ is in seconds $(s)$, displacement $x$ is in meters $(m)$, speed $\dot{x}$ is in $m / s$, and acceleration $\ddot{x}$ is in $m / s^{2}$. The above set up essentially means the following. Initially, both vehicles are stand still with the follower at $x_{i}(0)=0 \mathrm{~m}$ and the leader in front at $x_{j}(0)=5000 \mathrm{~m}$. At time $t=100 \mathrm{~s}$, a third vehicle in the adjacent lane traveling at $25 \mathrm{~m} / \mathrm{s}$ cuts in front of the follower $i$ at $x=2800 \mathrm{~m}$. As such, this third vehicle takes over as the leader $j$ and it keeps its speed constant up to $t=200 \mathrm{~s}$. Then, the leader begins to accelerate at a constant rate of $2 \mathrm{~m} / \mathrm{s}^{2}$ for 10 seconds, which results in an ending speed of $45 \mathrm{~m} / \mathrm{s}$. After this, the leader cruises at that speed up to $t=300 \mathrm{~s}$. Next, the leader applies a constant deceleration at a rate of $-3 \mathrm{~m} / \mathrm{s}^{2}$ for 15 seconds, which essentially brings the vehicle to a stop.

The following analysis assumes a normal driver who responds based on common sense. When the process starts, vehicle $i$ begins to move according to the logic stipulated in the microscopic model. Since vehicle $j$ is far away, it essentially has no influence on vehicle $i$ who is entitled to accelerate to its desired speed $\dot{x}_{i}(t) \rightarrow v_{i}=25 \mathrm{~m} / \mathrm{s}$. This process constitutes a free-flow regime. Right before $t=100 \mathrm{~s}$, the follower is at somewhere around $x=2745 \mathrm{~m}$ and the leader is $2255 \mathrm{~m}$ ahead. However, at $t=100 \mathrm{~s}$, the third vehicle cuts in right in front at $x=2800 \mathrm{~m}$ taking over as the new leader and shortening the inter-vehicle distance (i.e. spacing $s_{i j}$ ) to $55 \mathrm{~m}$. The sudden change in spacing causes the the follower to take emergency brake in order to maintain safe distance away from its leader. This process constitutes a braking regime. The emergency brake slows down the follower and the spacing between the two vehicles increases. When safe distance is achieved, the follower begins catch up with the leader's speed and maintain the safe distance thereafter. This constitutes a car-following regime. Starting from $t=200 \mathrm{~s}$, the leader begins to accelerate and eventually cruises at $45 \mathrm{~m} / \mathrm{s}$. As the leader speeds up, the spacing increases allowing the follower to accelerate as well. Since the leader travels much faster than the follower and the gap is opening, the follower is entitled to accelerate as desired and eventually settles at its desired speed $v_{i}=30 \mathrm{~m} / \mathrm{s}$. This returns to the free-flow regime again. Note that the leader's cruise speed is an exaggeration which is made on purpose to highlight the effect that the follower does not blindly follow its leader beyond its desired speed. At $t=300 \mathrm{~s}$, the leader begins to decelerate at comes to a stop at $x_{j}=10000 \mathrm{~m}$ after 15 seconds. As the follower approaches the stopped leader, the follower begins to decelerate, too, at a comfortable rate and finally rests right behind the leader. This process constitutes a transition from a approaching regime to the braking regime.

Figure 9 shows the performance of the special case formulated in Eq. (3.2). Three profiles are illustrated: displacement, speed, and acceleration. Broken red lines are for the leader whose motion is predetermined as above, while solid blue lines are for the follower whose motion is stipulated by the model. Examination of the follower's performances in the free-flow, approaching, car-following, braking regimes reveals that the model does conform to the above common sense analysis.

At the macroscopic level, the emphasis is to compare the simulated results against empirical observations across many vehicles and over time. The empirical data is collected from GA 400 by Georgia NAVIGATOR system. The resulting speed vs density, flow vs density, and speed vs flow plots are illustrated as dots in Figure 10. Solid blue lines show the performance of macroscopic special case (Eq. (3.4)) which is derived from Eq. (3.2) with the following parameters: free-flow speed $v_{f}=29$ 

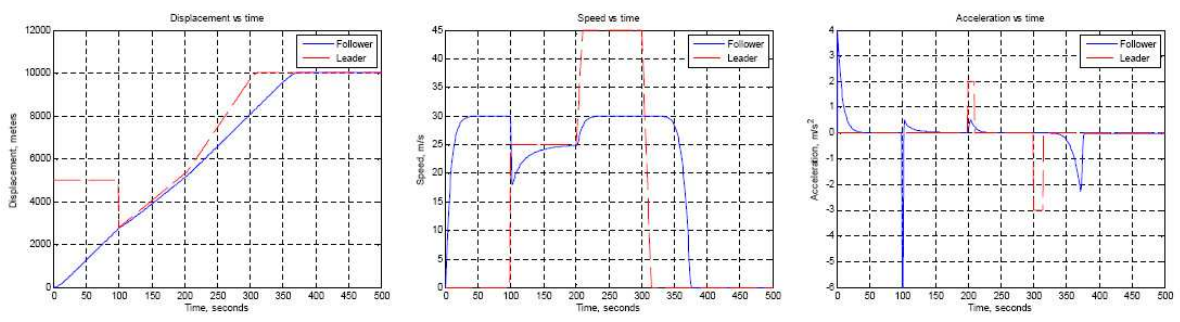

Figure 9: Performance of microscopic model special case (Equation 3.2)
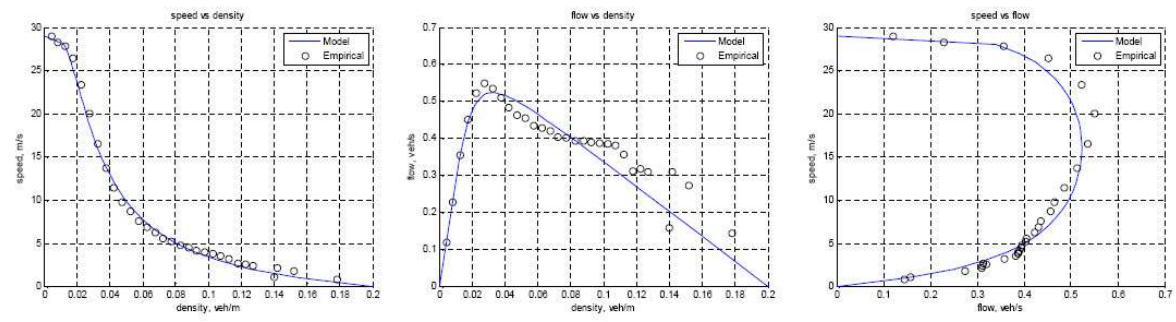

Figure 10: Performance of macroscopic model special case (Equation 3.4)

$\mathrm{m} / \mathrm{s}$, average perception-reaction time $=1.3 \mathrm{~s}$, and jam density $k_{j}=1 / 5 \mathrm{veh} / \mathrm{m}$. The plots show that the model agrees empirical observations very well.

\section{CONCLUSIONS}

This paper presents a broad perspective on traffic flow modeling at a spectrum of four scales: picoscopic, microscopic, mesoscopic, and macroscopic from the most to the least detailed level in that order. In order to ensure modeling consistency and provide a microscopic basis for macroscopic models, it is critical to address the coupling among models at different scales, i.e. how less detailed models are derived from more detailed models and, conversely, how more detailed models are aggregated to less detailed models. With this understanding, a consistent modeling approach is proposed based on field theory. Modeling strategies at each of the four scales are discussed. More specifically, the field theory serves as the basis of picoscopic modeling which represents a driver-vehicle unit as driver-vehicleenvironment closed-loop control system. The system is able to capture vehicle motion in longitudinal and lateral directions. The microscopic model is obtained from the picoscopic model by simplifying its driver-vehicle interactions, vehicle dynamics, and vehicle lateral motion. The mesoscopic model is derived from basic principles using the microscopic model as the mechanism of traffic evolution. The macroscopic model includes an evolution equation (which is derived by taking moments of the mesoscopic model) and an equilibrium speed-density relationship (which is the stationary solution to the mesoscopic model or derived from the microscopic model directly). Therefore, the proposed approach ensures model coupling and modeling consistency. A special case of the microscopic model is formulated. Further, its corresponding equilibrium speed-density relationships is derived. A numerical test is devised to verify if the microscopic special case makes any sense. In addition, the equilibrium relationship is validated against empirical data. Both numerical and empirical results suggest that the special case performs satisfactorily and aggregate to realistic macroscopic behavior.

\section{REFERENCES}

Abe, M. 1985. Theoretical Analysis on Vehicle Cornering Behaviours in Braking and in Acceleration. Vehicle System Dynamics 14 (1-3): 140-143. 
Ahmed, K., M. Ben-Akiva, H. Koutsopoulos, and R. Mishalani. 1996. Models of Freeway Lanechanging and Gap Acceptance Behavior. In Proceedings of the 13th International Symposium on the Theory of Traffic Flow and Transportation, 501-515.

Antoun, R., P. Hackert, M. O’Leary, and A. Sitchin. 1986. Vehicle Dynamic Handling Computer Simulation: Model Development, Correlation, and Application Using ADAMS. 12.

Ben-Akiva, M. E., M. Bierlaire, H. Koutsopoulos, and R. Mishalani. 1998. DynaMIT: A SimulationBased System for Traffic Prediction. DACCORS Short Term Forecasting Workshop.

Blaauw, G. J., H. Godthelp, and P. Milgram. 1984. Optimal Control Model Applications and Field Measurements with Respect to Car Driving. Vehicle System Dynamics 13 (2): 93-111.

Chandler, R., R. Herman, and E. Montroll. 1958. Traffic Dynamics: Studies in Car Following. Operations Research 6:165-184.

Chang, G.-L., T. Junchaya, and A. J. Santiago. 1994. A Real-Time Network Traffic Simulation Model for ATMS Applications: Part ISimulation Methodologies. Journal of Intelligent Transportation Systems 1 (3): 227241.

Eddie, L. 1963. Discussion on Traffic Stream Measurements and Definitions. In Proc. 2nd International Symposium of the Theory of Traffic Flow, Paris, France, 139154.

Gartner, N., C. J. Messer, and A. K. Rathi. 2001. Revised Monograph of Traffic Flow Theory: A State-of-the-Art Report. Transportation Research Board.

Gazis, D. C., R. Herman, and R. W. Rothery. 1961. Non-Linear Follow the Leader Models of Traffic Flow. Operations Research 9:545-567.

Gipps, P. 1981. A Behavioral Car Following Model for Computer Simulation. Transportation Research, Part B 15:105-111.

Gipps, P. 1986. A Model for the Structure of Lane-Changing Decisions. Transportation Research, Part B 20:403-414.

Hoogendoorn, S., and P. Bovy. 2001. State-of-the-art of Vehicular Traffic Flow Modelling. Proceedings of the IMechE Part I, Journal of Systems and Control Engineering 215 (4): 283-303.

Klar, A., and R. Wegener. 1999. A Hierarchy of Models for Multilane Vehicular Traffic (Part I: Modeling and Part II: Numerical and Stochastic Investigations). SIAM Journal on Applied Mathematics (SIAP) 59:983-1011.

LANL 1999. Transportation analysis simulation system (transims). Technical Report LA-UR-99-1658, Los Alamos National Laboratory.

Lighthill, M., and G. Whitham. 1955. On Kinematic Waves II. A Theory of Traffic Flow on Long Crowded Roads. Proc. Royal Society of London, Part A 229 (1178): 317-345.

Morrison, J., and V. Loose. 1995. TRANSIMS Model Design Criteria as Derived from Federal Legislation. Technical Report DOT-FTA-T-95-21, Los Alamos National Laboratory.

Ni, D. 2007. Determining Traffic Flow Characteristics by Definition for Application in ITS. IEEE Transactions on Intelligent Transportation Systems 8 (2): 181-187.

Ni, D. December 3-6, 2006. A Framework for New Generation Transportation Simulation. In Proceedings of Winter Simulation Conference '06. Portola Plaza Hotel, Monterey, CA.

Payne, H. 1971. Models of Freeway Traffic and Control. In Simulation Council Proceedings, Volume 1, 51-61.

Prigogine, I., and F. C. Andrews. 1960. A Boltzmann-like Approach for Traffic Flow. Operations Research 8 (6).

Raff, M., and J. Hart. 1950. A Volume Warrant for Urban Stop Signs. Technical report, Eno Foundation for Highway Traffic Control, Saugatuck, Connecticut.

Richards, P. 1956. Shock Waves on the Highway. Operations Research 4:42-51.

Smith, L., R. Beckman, D. Anson, K. Nagel, and M. Williams. 1995. TRANSIMS: Transportation Analysis and Simulation System. In Fifth National Conference on Transportation Planning Methods Applications-Volume II.

Tolman, R. C. 1980. The Principles of Statistical Mechanics. Dover Publications. 
Velan, S. M., and M. Van Aerde. 1996. Gap Acceptance and Approach Capacity at Unsignalized Intersections. ITE Journal 66 (3): 40-45.

Wegener, R., and A. Klar. 1996. A Kinetic Model for Vehicular Traffic Derived from a Stochastic Microscopic Model. Transport Theory and Statistical Physics 25:785-798.

Whitham, G. 1974. Linear and Nonlinear Waves. John Wileyand Sons Inc, New York, NY.

Wierwille, W., G. Gagne, and J. Knight. 1967. An Experimental Study of Human Operator Models and Closed-Loop Analysis Methods for High-Speed Automobile Driving. IEEE Transactions on Human Factors in Electronics HFE-8 (3): 187-201.

\section{AUTHOR BIOGRAPHIES}

DAIHENG NI is an Assistant Professor at the Department of Civil and Environmental Engineering, University of Massachusetts Amherst. He is a member of Transportation Research Board (TRB) Committee on Traffic Flow Theory \& Characteristics (AHB45) and a member of TRB Joint Simulation Subcommittee (AHB45(1)). His research interests are in traffic flow theory and simulation, Intelligent Transportation Systems (ITS), traffic sensing and information technology, and transportation logistics and optimization. His email address is <ni@ecs.umass.edu>. 